\title{
A Contrast of New Network Language Between Arabic and Chinese
}

\author{
Azza Abdelwahab Ibrahim Hassanein (Corresponding author) \\ Department of linguistics and applied linguistics, school of Chinese language and \\ literature, Wuhan University, Wuhan, Hubei province, China
}

Xun Zhai

Department of linguistics and applied linguistics, school of Chinese language and literature, Wuhan University, Wuhan, Hubei province, China

Received: October 9, 2019 Accepted: November 6, 2019 Published: November 8, 2019

doi:10.5296/elr. v5i2.15776

URL: https://doi.org/10.5296/elr.v5i2.15776

\begin{abstract}
Technology is a double-edged sword; the development and application of communication technologies have not only made great contributions to human beings and promoted the development of language, but also brought a rapid spread of language violence that caused great impact on people's lives. With the innovation of Internet technology, this form of language has developed rapidly in the dissemination of Internet media. At present, network language does not meet the requirements of language grammar, so there is a lack of teaching significance. Arabic and Chinese are subordinate to different language families with different network words. A prospective analysis has been performed between Arabic and Chinese network words which is mainly based on the influence of chivalrous network language on society and language. The research was based on 110 questionnaires distributed on social networking sites, and 40 interviews conducted with network users from china and some Arab countries. The findings suggested participants to use network language because, it is the language of their peers, it is cool and stylish, they have difficulties with the Arabic and Chinese language, and network constitutes a secret code, allowing escape from judgements of the older generation. The study concludes that network language is a strong marker of youth identity and group solidarity. Through a comparative analysis of Arabic and Chinese Internet words, Arabic or Chinese learners can clearly understand the similarities and differences between the two network words, and reduce the barriers to Internet communication, and appropriately use Chinese and Arabic Internet words.
\end{abstract}

Keywords: Internet, Network language, Arabic, Chinese, Differences, Similarities 


\section{Introduction}

While social networking sites (SNS) (Powale \& Bhutkar, 2013) are considered to be a form of communication, many aspects of our lives have been reconfigured and reshaped(Herring, 2008). An important aspect of these changes (SNS) is language (der Ham, Grosso, der Pol, Toonk, \& De Laat, 2007) (Antieau, 2009). One of the most common characteristics of the language is using in online communication platforms as web presentations, whether written or oral. As Crystal (2009) (Antieau, 2009) explains, network language has unique features that help users to write quickly and easily. This will simplify the online communication and create new features, such as the invention of new technical terms, acronyms including those associated with Internet use, such as acronyms used in text messages (Crystal \& others, 2001). Thus, it provides users with new opportunities to use the language. Internet users are able to create their own communication rules and adopt new rules that enable them to adapt to their voice communities (Baron, 2003).

\subsection{New Network Codes for Internet Users}

The popularity of social networking sites in the Arab world has resulted in a new writing code, Arabizi, which combines Roman letters and numbers to represent the Arabic language. This new code received vehement criticism from Arabic linguists who argued that Arabizi is detrimental to the Arabic language and Arab identity (Yaghan, 2008). The language used on the Arabian Web is called "arabizi" or "franko", It was developed by Arab users on social media sites and the Internet in the late 1990s in response to the need of using new technologies that do not yet support Arabic (Warschauer, Said, \& Zohry, 2002). Face book became a global site in 2006 and was published in English, French, German and Spanish. Although 250 million people use Arabic, it was not existed until 2009 when The Arabic version of The Black and Kiss was launched by Face book. Twitter was launched in 2006 and did not support Arabic until March 2012. Since its launch, Arabic has become "the fastest growing language ever on Twitter" (the influential Arab social media coverage of the Dubai Government College). In addition, Yahoo's 1997 launch of mail did not support Arabic scripts until 2009, including maktoob.com. Young people are often referred as the Internet generation, and use (Arabizi) on (SNS) as a means of communication.

\subsection{The Effect of Network Language on the Current Language}

Since 2006, China's Ministry of education and the State Language Commission have released the report on China's language living status to the community for five consecutive years. A total of 1176 Chinese neologisms have been published in the past four years (Zhao, Zhu, \& $\mathrm{Wu}, 2011)$. The average annual growth rate of new words is more than $25 \%$, which reflects the development and progress of society, economy and culture. The proportion of network vocabulary is more than $40 \%$. "From the perspective of language rules and usage characteristics, it is impossible for any language variant to be completely autonomous and closed (Yaghan, 2008), and to interact and penetrate with the universal language, especially at the lexical level. The network vocabulary cannot be equated with the network language, it is only the most active and fast-changing part of the network language. At present, there are several researches that focus on network language in general. David crystal discusses the linguistic repercussion of the internet in both its effect on the current language and the influence that might happen in the future (Antieau, 2009) Te Ma introduces the evaluation 
methods of online word-of-mouth effect from the perspectives of enterprises, consumers and third party websites, and expounds its significance (Ma, 2019). In 1994, Lazega EWasserman et al Covered some methods for the analysis of social networks and applied them to examples (Wasserman, Faust, \& others, 1994). In 2015 Freeman, Linton C reviewed the development of social network analysis from its earliest beginning until late 1990s, the history and the prehistory of social network analysis have been reviewed in that research(Freeman, 2011). In 2010 Solé et al examined the relevance of this trend for the study of human languages and review some early efforts to build up language networks, characterized their properties in addition to showing the developed direction models (Solé, Corominas-Murtra, Valverde, \& Steels, 2010). Other researches focused on arabizi which is a contemporary style of Arabic Slang (Yaghan, 2008). In 2018 Alghamdi et al performed a research on the use of arabizi in Saudi Arabia to figure out whether it is a deviant Form of Language or Simple Form of Expression (Alghamdi \& Petraki, 2018). While Keong et al explained The use of arabizi in English texting by Arab postgraduate students at UKM (Keong, Hameed, \& Abdulbaqi, 2015). However, several works mainly focus on internet language in china as ; 2016 liu lei et al who demonstrate the Rationality and Normalization of Chinese Network Words (Liu, 2016). In 2018 gao shen has done a research on the Principles and approaches of Network Words Standardization (Gao, 2018). While che fei made a Brief Introduction on the Research of Chinese Network Neologisms in the Past Ten Years (Che, 2015). Although many researches focused on the internet language and arabizi, there is a lack of any comparative researches between network language in Arabic and Chinese which is the main goal of this paper.

\section{Research Methodology}

This paper mainly includes the following methods:

\subsection{Document Survey Method}

To investigate the research results of the previous researches by using textbooks and referring to relevant literature and theoretical works for the means of gaining sufficient study references.

\subsection{The Contrastive and the Comparative Method}

Based on its research ideas and research methods, this paper makes a contrastive analysis of Arabic and Chinese Internet words, and expounds the similarities and differences between both Arabic and Chinese network words respectively from the types and structures of words. Within deep research on the social culture, the reason that network language existence has been figured out and thus leads to further approaches.

\subsection{Questionnaire}

The research questionnaire was based on 110 questions distributed on social networking sites, most of the e-questions were completed in Arabic or Chinese, except for 8 high school students, who answered the e-questionnaire in English and/or chatroom language. The e-questionnaire respondents were between 18 to 30 years old, including $25 \%$ males and $75 \%$ females. The majority of respondents who completed the e-questionnaire $(63.3 \%)$ were between 18 to 22 years old and the rest (37.7\%) ranged between 23 to 30 years old.

\subsection{Interview}

An interview has been done for 40 persons; among them 20 Arab, and 20 Chinese based on 
the following selection criteria:

-Half of them is young people age between 18-30 years old, and the others is above30years old.

-All of them should be familiar with social media.

The interview was mainly aiming to figure out the reason for using network language, what are their motivations behind the use of network language, and What are the attitudes and beliefs of young users towards network language.

\section{Results and Findings}

The findings are organized based on the research questions, and combine frequently reported themes from the e-questionnaire and interview responses.

\subsection{Attitudes and Beliefs About Network Language}

Network language users reported a variety of attitudes towards and beliefs about this language. The results from the e-questionnaire and the interview were similar but, in the interviews, additional information was offered, due to the nature of the interviews. The most frequently cited attitudes are (1) network language is a code that is used for informal communication; (2) network language is easier and faster than Arabic and Chinese in terms of grammar and writing; (3) network language is described as cool, stylish, trendy, and the language of young people on social media; (4) network language may have some negative effects on the participants' Arabic and Chinese language writing skills, and (5) network language does not undermine the Arab and Chinese culture and/or identity. Figure 1.

\subsection{Network Language Informality}

Based on the e-questionnaires and the interviews, the majority of network language users participating in this study indicated that this language is an informal code. They explained that they use the script to chat with their friends and relatives within their age group on SNSs. However, they would not use this language in formal settings, such as communicating with their boss at work or send a formal letter. Figure 1.

\subsection{Network Language Flexibility}

Network language is easier and faster than Arabic and Chinese especially for online communication. It was reported that network language lacks spelling conventions, while their mother language script has strict grammatical rules and diacritics. Network language provides them with an opportunity to hide their poor language skills and an escape from certain grammatical/spelling rules when writing in Arabic or Chinese script. Figure 1.

\subsection{Network Language Feedback}

It is considered as Cool, Stylish, and Trendy; the participants viewed it as a writing style which defines their young, playful, and flexible nature as young people. Using network language is a form of expression on SNSs and a form of solidarity among youth. it guarantees their belonging to a group of stylish, fresh, and trendy young people. Figure 1 .

\subsection{Network Language Negative Effects}

Although most network language users reported their favorable attitude towards this language, some of them acknowledged the possible negative effects that may affect their Arabic or Chineses kills due to the widespread negative propaganda against network language in China 


\section{Macrothink}

and Arabic societies. A lot of the participants admitted the possible negative effect of this language on their mother language skills. Figure 1.

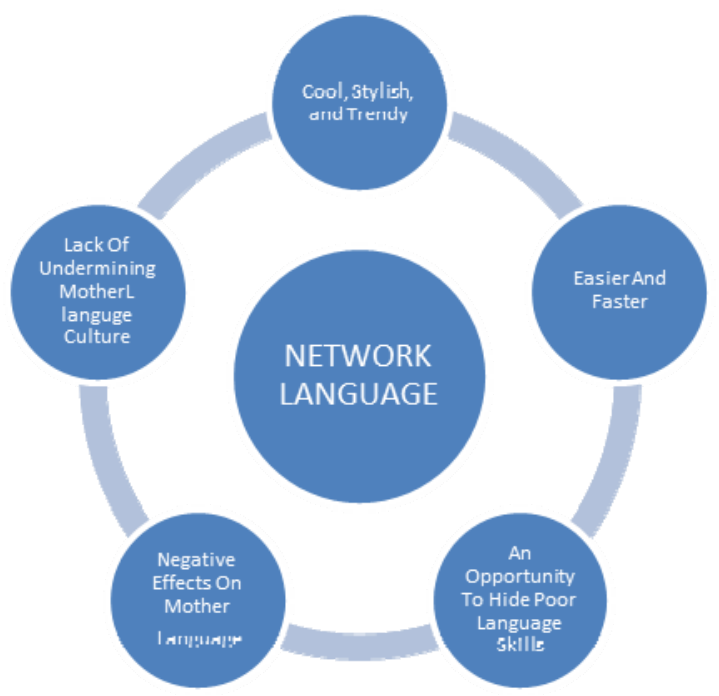

Figure 1. Network language advantages and disadvantages

\section{Discussion}

\subsection{Classification of Arabic and Chinese Internet Words}

One of the most prominent features of network language is the existence of Internet-specific vocabulary. There are five main types of network languages: World Wide Web Language, E-mail Language, Network Chat Language, Virtual World Language and Blog Language. This variant is mainly formed with the development of Internet technology and the expansion of mobile phone business. With the further development of network technology, the diversification of network terminal forms and the variation of network language will be enhanced.

\subsubsection{The Classification of Arabic Network Words}

Network language is a new form of language, and chat room language is one of them. With the popularity of the Internet, the increase in the number of Internet users and the advantages of the Internet itself has become an indispensable communication tool for modern people. People can carry out various activities through the network, which greatly enriches people's spiritual world, as the carrier of network communication.

Arabic network words are divided into: chat room language, World Wide Web language, e-mail language and instant messaging language.

I. Chat room language; Chat room language is the most threatening language in Arabic. The main reason that people use English to write Arabic in chat rooms is the lack of its support by the software which allows non-English-speaking people to write Arabic with English letters (Al-Khatib \& Sabbah, 2008). The language is called "franko" (Warschauer et al., 2002). And people also create new ways of writing letters that do not exist or have the same pronunciation in English, such as transferring Arabic letters to numbers. 
II. English letters instead of Arabic letters; such as $=J, N=\dot{U}, F=\dot{\theta}, D=د$ etc.

Table 1. Some examples of Franko words and their meaning

\begin{tabular}{lll}
\hline ARABIC WORDS & FRANKO & THE MEANING \\
\hline السلام عليكم & Al salamualikoum & Hello \\
شكرا جزيلا & Shokranjazilan & Thank you \\
كيف حالك؟ & Kaifhaloka $?$ & How are you? \\
\hline
\end{tabular}

III. Arabic letters that do not exist in English; In order to chat in Arabic on software that does not support Arabic, Arabs try to deal with Arabic that does not have the same pronunciation in English, so Arabs use numbers to express these letters, for example:

$\varepsilon=3, \stackrel{ث}{=}=4$ etc. .

Table 2. Some numbers expressing Arabic letters which are not existed in English

\begin{tabular}{|c|c|c|c|c|}
\hline Arabic letter & Number & Arabic word & Franko & The meaning \\
\hline$\varepsilon$ & 3 & عربي & 3raby & Arabic \\
\hline همزه (ء) & 2 & سؤال & So2al & Question \\
\hline 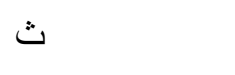 & 4 & ثلاجه & 4alaga & Fridge \\
\hline$\dot{\varepsilon}$ & 3 ' & غد & 3'ad & Tomorrow \\
\hline
\end{tabular}

IV. World Wide Web Language; As some software and equipment do not support Arabic filenames, this allows users to use Latin letters to write Arabic words and English to borrow Arabic meanings (Dorogovtsev \& Mendes, 2001). As an example, the names of these websites are all Arabic names, but they are all written in Latin letters:

- www.alfaseeh.com, مكتبه الفصيح

- www.alquds.edu, امعه القدس

- $\quad$ www.ahlalhadeeth.com, ملتقي أهل الحديث

V. Arabish or Arabizi language; This language is a combination of Arabic and English (Yaghan, 2008). It refers to Arabic written informally in Latin letters and usually used in computers or mobile phones that do not support Arabic, for example.

- Changing some English idioms into Arabic to borrow the English meaning (bilingual expression) (Alanazi, 2018), such as: Check the e-mail = شيك علي الميل كيل كنسل المو عد

- Using English idioms (use Alphabetic letters to write English idioms), for example;

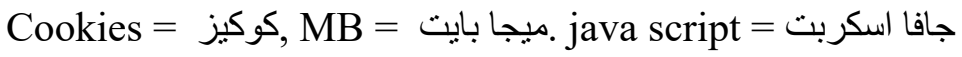

- Using Abbreviated English letters, one way of writing is to abbreviate English idioms, for example; $\mathrm{TC}=$ take care, $\mathrm{LOL}=$ laughing out loud, $\mathrm{BRB}=$ be right back 


\subsubsection{Classification of Chinese Internet Words}

Network language is a kind of language produced or applied in network communication (Che, 2015), including English letters, punctuation, symbols, pinyin, icons (pictures) and text and other combinations. This combination often expresses its special significance in the specific network media communication.

Chinese network words are divided into;

I. Chat Room language; Chat room language refers to the language commonly used by netizens in social media ( $\mathrm{Li} \& \mathrm{Xu}, 2016$ ). It is different from the network terminology and the special terminology on the network as it refers to the language used to chat and express one's own opinions and feelings. In addition to general words, such as "I flash" and "Dong Dong", it also includes special symbols, numbers, letters and emoticons, such as 520 (I love you), IC (I see), GX (congratulations), etc.

-Language features of Internet chat rooms are as follows:

*Conciseness; mainly by text input, simplicity is mainly reflected in abbreviations, such as me2 (me too), MM (sister), 886 (bye-bye), CM (smelly beauty), F2F (face to face), BD (fool), ILV (I love you), 837 (don't get angry).

*Arbitrariness; the randomness is manifested in the mixing of Chinese and English, the mixing of symbols and letters, etc. 如有事情 call 我、我每天都在 home, It means to call me when something happens, I'm at home every day. Another example, (3Q\&CU), which means thank you. See you next time.

*Symbolism; Symbols are used to express feelings, such as I $<3$ you (I love you), ^.^ (girl laughs), $\wedge \wedge$ (boy laughs). There are some more complex and vivid emoticons, such as $\sim @ \wedge$ @ (lovely)(Wasserman et al., 1994).

*Innovativeness; for example, $456=$ me, you have wood $=$ whether or not, shrimp $=$ online master, $356=$ online, $6785753=$ the old place is not scattered etc.

II. Blog language; It is done by a person who publishes and posts personal articles on the Internet using specific software, or a website that is usually managed by individuals and posts new articles on an irregular basis (Bolander, 2013). By comparing Chinese and foreign blog languages, we can find that although the specific phenomena of blog languages in different countries are different, they have more common characteristics. Articles are usually displayed in reverse chronological order, and various types of discourse are arranged between the two poles (Bolander, n.d.). For example, daily dialogues (a) expressed by the same voice are more colloquial than academic reports (b), while newspaper reports (c) expressed by the same writing style are closer to the pole of written voice than personal letters (d). The latest article first appeared at the top of the page.

III. Bilingual expressions in Chinese and English; There are two kinds of Chinese-English bilingual expressions, one is the direct English expression, such as BB (baby), and the other is the transfer of Chinese meaning, referring to the use of English words as Chinese words (Gao, 2018). Chinese expresses modesty by saying "where, where 哪里, 哪里". If you praise a netizen on the Internet "you are so smart", he can answer "where, where 哪里, 哪里". They say "a trivial matter" as "Xiao case", and "if you need something call me, e-mail" as "if you need something call, E-me 有事 call、E 我". However, it is worth noting that this kind of 
English not only borrows the meaning of Chinese, but also follows the expression sentence pattern of Chinese, and is incorporated into the expression system of Chinese (Yang, 2009). English-Chinese translation refers to meaningful transliterated words, that is, meaningless English pronunciation and writing meaningful Chinese words, and according to such Chinese characters. For example, E-mail writing "伊妹儿", HOME PAGE writing "烘焙鸡", MODEM writing "猫", DOWNLOAD writing "荡" etc.

\subsection{The Way of Word Formation in Arabic and Chinese Network Words}

\subsubsection{The Way of Word Formation in Arabic Network Words}

Through the classification of Arabic network words, it has been found that the main components and expressions of Arabic network words mainly include the following eight ways: homophonic word formation, abbreviation, homophonic transliteration of foreign language words, digital homophonic words, various non-literal symbols, direct borrowing of English words , English words or phrases and abbreviation of acronym (Warschauer et al., 2002);

I. Direct borrowing of English vocabulary; It is the most frequently used method of Arabic network language, such as (hi = ررحب ), (SMS = رساله نصيه ) etc. .

II. Abbreviations; The abbreviated word-formation method makes the network vocabulary structure concise and easy to use, and meets the requirements of efficient and rapid information exchange. There are two ways to abbreviate Arabic net language:

- Word abbreviation Keep only affixes or roots in words. For example: Tel (telephone) etc .

- Phrases and sentence abbreviations; (Al-Khatib \& Sabbah, 2008)Connect phrases with the first letter of each word in a sentence, for example: AA (al salamu alikoumast (السلام عليك), Isa (in shaa allaha ان شاء اللهز الك الله خيرl) , JAK (jazak allah khairan) BRB (be right back)、 tyt(take your time)etc.

III. Homophonic word formation, It refers to the use of the same or similar vocabulary pronunciation to construct new vocabulary through the following two methods;

-According to the pronunciation characteristics of the words and the combination of other words into simplified network neologisms, such as: $\mathrm{Cu}$ (see you), U2 (you too), W8 (wait).

-Replace words with letters with the same pronunciation, such as IDK (I don't know), ASAP (as soon as possible).

IV. Hybrid Form of Letter and Number. For example: 2alby = حاضر = قو ال = etc.

Various non-verbal symbolic methods; For example:(:): etc.

VI. Homophonic Transliteration of Foreign Language Vocabulary; This is common in Arabic to find suitable letters for replacement, such as: e-mail = ايميل.

\subsubsection{The Way of Word Formation of Chinese Network Words}

Recently, network language has had a great impact on Chinese. The author collected and classified the vocabulary of "100 common network vocabulary occurrence frequency, usage and control list" and 128 vocabulary acquired through network practice (Zheng, 2016). The author finds that the method of word formation of Chinese network vocabulary is similar to 
that of Arabic network vocabulary, including 7 kinds of Chinese Pinyin abbreviation grammar, abbreviation and digital homophonic words (Zhang, 2013).

-Chinese Pinyin abbreviation. For example: $G G=$ 哥哥, $J J=$ 姐姐, $\mathrm{DD}=$ 弟弟, $\mathrm{ZT}=$ 转 etc.

-Direct borrowing of English words. For example: fans = 粉丝 etc.

-Hybrid Form of Letter and Number; for example: F2F (Face to Face) 、3Q (thank you) 、 me2 (me too) etc. .

-Digital homophonic method; By using numbers instead of words in network chat, for example :065 =原谅我,

$837=$ 你别生气, $918=$ 加油吧, $687=$ 对不起 etc.

-Character Symbol Method; combining a number of simple graphic symbols, it uses freehand brushwork to delineate certain characteristics of people or things, and depicts a certain mood of people, which has strong visual and rhetorical effects, for example:

$\wedge_{-} \wedge=$ 高兴, :): $=$ 微笑 etc.

-Shortening; Abbreviations are compressed mainly by morpheme extraction, there are also some Chinese abbreviations which use the phonetic similarity between words to abbreviate two pronunciations into one, for example: 表(不要)、宣(喜欢)etc.

-Homophonic Transliteration of Foreign Language Vocabulary; According to the pronunciation of loanwords to find suitable Chinese characters to replace them, such as mouse (鼠标), e-mail(伊妹儿)etc.

\subsection{Common and Different Word-building Methods in Arabic-Chinese Network Language}

Although Arabic and Chinese belong to different language families, Arabic is phonetic, while Chinese is ideographic (phonetic-ideographic combined text), there must be many differences in the development of the two languages, but from the comparative analysis of network languages, it is found that Arabic and Chinese network languages have the same way of word formation but in different ways. The similarity is that the network language communication environment determines that the Arabic-Chinese network language is based on economic principles, which significantly abbreviates the different language systems of Arabic and Chinese. The Arabic-Chinese network language is arbitrary in word formation and has the phenomenon of endowing old words with new meanings.

\subsubsection{The Common Points of Arabic and Chinese Network Language Formation Methods}

-Abbreviation of Arabic-Chinese Network Language; One of the major characteristics of the Internet age is time-saving in addition to the efficient and fast-paced network communication to achieve the goal of economic saving. Therefore, in online chat, we must use fewer characters to express meaning, improve the rhythm and efficiency of online communication. In addition, these abbreviations are easy to remember and use. Therefore, there is a trend of word simplification in both English and Chinese network languages, which is manifested in the use of abbreviations and symbols in word formation, for example in Chinese GG (哥哥), and in Arabic AA (السلام عليكم).

-The arbitrariness of Arabic-Chinese network language; Arabic-English network neologisms are random and occasional in word formation. Everyone can create his own network words 
on the internet. Some people create their own words from the perspective of Pinyin and others create their own words from the perspective of homophonic, for example: in Arabic $\mathrm{KK}=$ okay, and in Chinese okay $了=$ okay. Moreover, there is a general trend of arbitrary dismemberment of traditional words on the English network, that is, deliberately using created words instead of using inherent words, and deliberately misusing grammar. At the same time, there are also some new words which are short-lived and cannot be spread.

-The New Meaning appearance of Old Words in Arabic-Chinese Network Language; expanding the meaning of words is also a very important way to produce new words, because the current vocabulary is not enough relative to the things and concepts we need to express. There are many new meanings of old words in both languages. Such as cookies in Arabic, and dinosaurs 恐龙 in Chinese, etc. "Cookies" was originally meant to be biscuits, the new meaning is a new technology that allows Web servers to store or read data. 恐龙 (dinosaurs) Originally refers to large extinct reptiles, the new meaning is ugly female netizens.

-The Use of Loanwords in Arabic and Chinese Network Languages; Arabic and Chinese network languages use a lot of foreign loanwords, for about 32\%. Arabic network languages have some loanwords. Most of these loanwords come from countries with more developed network culture, such as Italian Word Ciao (goodbye), viva (ten thousand). In addition, the Chinese wangba (王八) has also been absorbed into the English network language.

\subsubsection{The Differences Between Arabic and Chinese Network Word Formation French}

Although there are many commonalities in the word-formation of Chinese and Arabic network neologisms, there are obvious differences in the word-formation of Chinese and Arabic network neologisms due to the differences in the development stages of network technology, as the two languages belong to two different language families.

-There are many partials words in Chinese network language; Arabic network language has no way of word formation in Chinese network neologisms. The most prominent part of Chinese network neologisms is a large number of homophonic words, which account for $18.1 \%$ of Chinese network neologisms. For example, Chinese has a large number of numbers used that express a commonly used phrase. For example, 520 (I love you 我爱你), 886 (bye bye 再见), etc.

- Synonyms in Chinese and Arabic Network Language; There are many synonyms in Chinese network language; one prominent phenomenon of Chinese network neologisms is the emergence of synonyms, such as "hacker" 黑客 and "hacker" 骇客. While Arabic has no tone, Therefore, homonym is not as prominent as Chinese. Arabic has "tashkeel" (Marking as an alphabetical guide), So the ability to express meaning in isolation is stronger than that in Chinese.

-Chinese syllables are relatively simple, mainly through tones to distinguish the meaning of words, however there is no tone in the Chinese character input method, so many synonyms also appear. Netizens typed these homonyms without choosing, and over time they were gradually accepted and used. While Arabic, as a phonetic language, has no homophonic synonyms in cyber Arabic.

-Usage of mother language letters in network language; regarding the Arabic network language, users do not use any Arabic letters but only use the Latin words as the Arabic 
letters could not make any difference in the network language, while in the Chinese network language, there are some abbreviations in Chinese that users always depend on.

\section{Conclusion}

Through a comparative analysis of Chinese and Arabic Internet words, use the Arabic and Chinese Internet words. It has been found that there are similarities and differences which greatly enriched the word formation methods of network words and promoted the comparative development of Chinese and Arabic. This paper based on questionnaire, which included 110 questions distributed on social networking sites, the e-questionnaire respondents were between 18 to 30 years old, and an interview has been done for 40 persons; among them $20 \mathrm{Arab}$, and 20 Chinese. Which mainly aiming to figure out the reason for using network language, what are their motivations behind the use of network language, and What are the attitudes and beliefs of young users towards network language. This research has provided substantial insights into network language use by young people. It has paved the way for extending and enhancing the understanding of network language, its use, and its future.

\section{References}

Al-Khatib, M., \& Sabbah, E. H. (2008). Language choice in mobile text messages among Jordanian university students. SKY Journal of Linguistics, 21(1), 37-65.

Alanazi, M. M. J. (2018). Exploring the Function and Status of 'Arabish': A Critical Study of Class Distinction in Informal Saudi Instant Message Interactions. King's College London.

Alghamdi, H., \& Petraki, E. (2018). Arabizi in Saudi Arabia: A Deviant Form of Language or Simply a Form of Expression? Social Sciences, 7(9), 155. https://doi.org/10.3390/socsci709 0155

Antieau, L. (2009). Language and the Internet, by David Crystal. https://doi.org/10.1080/ 15427580903121519

Baron, N. S. (2003). Language of the Internet. The Stanford Handbook for Language Engineers, 59-127.

Bolander, B. (n.d.). Language and Power in Blogs: Interaction, Disagreements and Agreements. Pragmatics \& Beyond New Series, vol. 237. John Benjamins, Amsterdam.

Bolander, B. (2013). Language and power in blogs: Interaction, disagreements and agreements (Vol. 237). John Benjamins Publishing Company. https://doi.org/10.1075/pbns. 237

Che, F. (2015). In the past ten years, the research of Chinese network neologisms is summarized. Journal of Chongqing University of Technology (SOCIAL SCIENCES), 102-113.

Crystal, D., \& others. (2001). Language and the Internet. Cambridge University Press. https://doi.org/10.1017/CBO9781139164771

der Ham, J., Grosso, P., der Pol, R., Toonk, A., \& De Laat, C. (2007). Using the network description language in optical networks. 2007 10th IFIP/IEEE International Symposium on Integrated Network Management, 199-205.

Dorogovtsev, S. N., \& Mendes, J. F. F. (2001). Language as an evolving word web. Proceedings of the Royal Society of London. Series B: Biological Sciences, 268(1485), 


\section{Mll Macrothink \\ Education and Linguistics Research \\ ISSN 2377-1356 \\ 2019, Vol. 5, No. 2}

2603-2606. https://doi.org/10.1098/rspb.2001.1824

Freeman, L. C. (2011). The development of social network analysis--with an emphasis on recent events. The SAGE Handbook of Social Network Analysis, 21(3), 26-39. https://doi.org/ 10.4135/9781446294413.n3

Gao, S. (2018). A study on the principles and approaches of the standardization of network words. Literature Education

Herring, S. C. (2008). Language and the Internet. The International Encyclopedia of Communication.

Keong, Y. C., Hameed, O., \& Abdulbaqi, I. A. (2015). The use of Arabizi in English texting by Arab postgraduate students at UKM.

Liu, L., Lou, B., \& Li, G. T. (2016). The rationality and standardization of network words. Chinese construction, 1Z, 97.

Li, Y. N., \& Xu, J. H. (2016). An investigation and analysis of Japanese source word recognition in Chinese network words. Journal of Beijing University of Posts and Telecommunications: Social Sciences, 4, 101-107. https://doi.org/10.3139/146.016022

Ma, T. (2019). Evaluation effect of Internet word of mouth and application of big data. Journal of Physics: Conference Series, 1187(5), 52050. https://doi.org/10.1088/1742-6596/ $1187 / 5 / 052050$

Powale, P. I., \& Bhutkar, G. D. (2013). Overview of privacy in social networking sites (sns). International Journal of Computer Applications, 74(19). https://doi.org/10.5120/13005-0311

Solé, R. V., Corominas-Murtra, B., Valverde, S., \& Steels, L. (2010). Language networks: Their structure, function, and evolution. Complexity, 15(6), 20-26. https://doi.org/10.1002/cplx.20305

Warschauer, M., Said, G. R. El, \& Zohry, A. G. (2002). Language choice online: Globalization and identity in Egypt. Journal of Computer-Mediated Communication, 7(4). https://doi.org/ 10.1111/j.1083-6101.2002.tb00157.x

Wasserman, S., Faust, K., \& others. (1994). Social network analysis: Methods and applications (Vol. 8). Cambridge University Press.

Yaghan, M. A. (2008). “Arabizi”: A contemporary style of Arabic Slang. Design Issues, 24(2), 39-52. https://doi.org/10.1162/desi.2008.24.2.39

Yang, Q. X. (2009). Comparison of word formation in English and Chinese network languages.

Zhao, Y., Zhu, Q., \& Wu, K. (2011). The development of social network analysis research in mainland China: a literature review perspective. Proceedings of the 2011 I Conference, 296-303.

Zhang, Y. F. (2013). the word formation of Chinese network neologisms. Chinese teaching communication: learning (D), 7, 82-83.

Zheng, Z. Y. (2016). On the word formation of Chinese network catchwords. Journal of Lanzhou Institute of Education, 32(10), 61-62. https://doi.org/10.1055/s-0036-1583228. 


\section{Macrothink

\section{Copyright Disclaimer}

Copyright reserved by the author(s).

This article is an open-access article distributed under the terms and conditions of the Creative Commons Attribution license (http://creativecommons.org/licenses/by/3.0/). 